\title{
miR-106b modulates cancer stem cell characteristics through TGF- $\beta /$ Smad signaling in CD44-positive gastric cancer cells
}

\author{
Dayeon $\mathrm{Yu}^{1,2}$, Hyun-Soo Shin ${ }^{3,4}$, Yeo Song Lee ${ }^{1,2}$ and Yong Chan Lee ${ }^{1,2}$
}

Cancer stem cells have the capacity to form new tumors and are thus considered to be a cause of metastasis and tumor recurrence. However, many of the mechanisms determining cancer stem cell characteristics are still unknown. MicroRNAs (miRNAs) are possible modulators of cancer stem cell generation and may be involved in the retention of cancer stem cell characteristics. The aim of this study was to examine the miRNA expression profiles regulating the cancer stem-like cell characteristics in gastric cancer. We sorted gastric cancer stem-like cells using the stem cell marker CD44 by fluorescenceactivated cell sorting. CD44( + ) cells formed more and larger spheres compared with CD44( -$)$ cells. Cancer stem cell markers were overexpressed in CD44(+) cells. CD44(+) cells showed increased expression of mesenchymal cell markers, whereas epithelial markers were downregulated. In miRNA microarray, the miR-106b family comprising miR-106b, miR-93, and miR-25 was significantly upregulated in CD44(+) cells than in CD44( -$)$ cells. Smad7, which inhibits transforming growth factor- $\beta$ (TGF- $\beta$ )/Smad signaling as a target of the miR-106b family, was downregulated in CD44( + ) cells.

Furthermore, expression of TGF- $\beta /$ Smad signal molecules was activated in CD44 $(+)$ cells, in accordance with the action of the miR-106b family. Inhibition of miR-106b showed suppression of the TGF- $\beta /$ Smad signaling pathway and decreased self-renewal capacity and cell invasiveness. Our study suggests that CD44(+) gastric cancer cells show cancer stem cell properties with epithelial-mesenchymal transition (EMT). Increased miR-106b family expression regulated cancer stemlike cell properties, particularly EMT characteristics, through the TGF- $\beta /$ Smad signaling pathway in CD44( + ) stem-like cells. Taken together, these results indicate that targeting miR-106b may be an effective form of cancer therapy in gastric cancer through the modulation of cancer stem cell characteristics.

Laboratory Investigation (2014) 94, 1370-1381; doi:10.1038/labinvest.2014.125; published online 6 October 2014

Gastric cancer has the second highest mortality rate among cancers and is the fourth most common cancer in the world. ${ }^{1}$ Helicobacter pylori infection and dietary factors are thought to be the main causes of this disease. ${ }^{2}$ Cancer stem cells are known tumor initiating cells. They have self-renewal properties, the potential to differentiate into any cells in the tumor population and considerable proliferative capacity. ${ }^{3}$ Also, cancer stem cells are considered to be major causes of tumor metastasis and recurrence because of their mesenchymal behavior.

Cancer stem cells have been identified in breast, brain, and several other cancers including gastric cancer. ${ }^{4-8}$ One of the well-known techniques to fractionate cancer stem cells employs several surface markers overexpressed in cancer stem cells. CD44 is a transmembrane glycoprotein widely used to isolate cancer stem cells in various cancers, particularly in gastric cancer. ${ }^{9}$

Epithelial-mesenchymal transition (EMT) has an important role in embryonic development in many animals. In the EMT process, epithelial cells lose their characteristics and then take on mesenchymal characteristics. EMT is considered a possible target of anti-cancer therapy because it confers invasive and metastatic ability on cancer cells. ${ }^{10}$ In addition, EMT shares several properties with cancer stem cells. A study showed that a fraction of CD $44^{\text {high }} / \mathrm{CD} 24^{\text {low }}$ cancer stem-like cells were increased when EMT was induced in mammary epithelial cells. ${ }^{11}$

\footnotetext{
'Department of Internal Medicine, Institute of Gastroenterology, Yonsei University College of Medicine, Seoul, Korea; ${ }^{2}$ Brain Korea 21 PLUS Project for Medical Science, Yonsei University College of Medicine, Seoul, Korea; ${ }^{3}$ Division of Nephrology, Department of Internal Medicine, Ewha Medical Research Center, Seoul, Korea and ${ }^{4}$ Ewha Womans University School of Medicine, Ewha Medical Research Center, Seoul, Korea

Correspondence: Professor YC Lee, MD, Department of Internal Medicine, Institute of Gastroenterology, Yonsei University College of Medicine, 134 Shinchon-Dong, Seodaemun-Ku, Seoul 120-752, Korea.
}

E-mail: leeyc@yuhs.ac

Received 9 January 2014; revised 1 September 2014; accepted 3 September 2014 
MicroRNA (miRNA), a small non-coding RNA ( 22nt), has drawn attention in recent years as one of the key modulators of cellular properties. A miRNA network is a complicated system and its dynamic interaction with various targets regulates many cellular processes by altering gene expressions through cleavage of target mRNA, translational repression, and mRNA deadenylation. ${ }^{12}$ Also, miRNA may be an important regulator of cancer stem cell properties. miR200c suppresses cell differentiation by modulation of bmil in breast cancer cells ${ }^{13}$ and miR-34a represses generation of prostate cancer stem cells. ${ }^{14}$ The miR-106b family is overexpressed in several tumors, including gastric cancer. ${ }^{15-18}$ The miR-106b family is implicated in many cellular signaling pathways: regulation of cell cycle through $\mathrm{p} 21^{19}$ and activation of the transforming growth factor- $\beta$ (TGF- $\beta$ ) signaling pathway. ${ }^{20}$

Herein, we have identified cancer stem cell phenotypes in $\mathrm{CD} 44(+)$ stem-like cells in gastric cancer. We then analyzed the miRNA expression profiles between $\mathrm{CD} 44(+)$ and $\mathrm{CD} 44(-)$ cells to evaluate miRNA profiles responsible for modulating gastric cancer stem cell characteristics. We found that upregulated miR-106b-activated TGF- $\beta /$ Smad signaling modulates stem cell characteristics in $\operatorname{CD} 44(+)$ gastric cancer stem-like cells.

\section{MATERIALS AND METHODS Cell Culture}

MKN45 (KCLB 80103) and KATO III (KCLB 30103) gastric cancer cells were maintained in RPMI-1640 medium (Thermo Scientific, Rockford, IL, USA) supplemented with $10 \%$ FBS (Thermo Scientific) and $1 \%$ penicillinstreptomycin sulfate (Thermo Scientific). SW480 (KCLB10228) colon cancer cells were maintained in DMEM medium (Thermo Scientific) supplemented with 10\% FBS (Thermo Scientific) and 1\% penicillin-streptomycin sulfate (Thermo Scientific).All cultures were maintained in a $37^{\circ} \mathrm{C}$ incubator supplemented with $5 \% \mathrm{CO}_{2}$.

\section{Flow Cytometry Analysis and Fluorescence-Activated Cell Sorting}

For flow cytometry, about $80 \%$ confluent cells in a $100-\mathrm{mm}$ cell plate were washed with PBS and then cells were detached from plates using Trypsin-EDTA and centrifuged at $4{ }^{\circ} \mathrm{C}$. Cell pellets were resuspended in HBSS (Gibco, Grand Island, NY, USA) supplemented with $1 \mathrm{mM}$ HEPES (Gibco) and $2 \%$ FBS and filtered with a $40-\mu \mathrm{m}$ mesh filter (BD Biosciences, San Jose, CA, USA). The cells were stained with a 400-fold dilution of anti-CD44-FITC (BD Biosciences) and incubated for $30 \mathrm{~min}$ in the $37^{\circ} \mathrm{C}$ incubator supplemented with $5 \% \mathrm{CO}_{2}$. Then the cells were washed with HBSS and resuspended in HBSS supplemented with $1 \mathrm{mM}$ HEPES, $2 \%$ FBS, and $1 \%$ penicillin-streptomycin sulfate. They were then analyzed and sorted immediately with fluorescence-activated cell sorting (FACS) AriaIII (BD Biosciences).

\section{Sphere Colony Formation Assay}

Gastric cancer cells were planted in each well of ultra-lowattachment 24-well plates (Corning Life sciences, Corning, NY, USA) with DME/F12 medium (Thermo Scientific) supplemented with $20 \mathrm{ng} / \mathrm{ml}$ EGF (R\&D Systems, Minneapolis, MN, USA), $10 \mathrm{ng} / \mathrm{ml}$ basic FGF (R\&D Systems), 1\% ITS (Gibco), and 1\% penicillin-streptomycin sulfate. Every 3 days, each well was examined using light microscopy.

\section{Real-Time PCR}

Total RNA was extracted from culture cells using TRIzol reagent (Invitrogen, Carlsbad, CA, USA) according to the manufacturer's instructions. First-strand cDNA was synthesized using oligo(dT) primers and Superscript II reverse transcriptase (Invitrogen). Real-time PCR was carried out with a PCR mixture containing $1 \mu \mathrm{mol} / \mathrm{l}$ of each primer and SYBR Green master mix (Applied Biosystems, Foster City, CA, USA). The amplifications were conducted at $95^{\circ} \mathrm{C}$ for $10 \mathrm{~s}$ and at $60^{\circ} \mathrm{C}$ for $60 \mathrm{~s}$ using a StepOnePlus real-time PCR system (Applied Biosystems). Each sample was examined in triplicate and the amount of PCR product was normalized with respect to $\beta$-actin as an internal control. PCR primers are shown in Table 1.

cDNA for miRNA assay was synthesized using the TaqMan MicroRNA Reverse Transcription Kit (Applied Biosystems) with miR-106b-, miR-93-, miR-25-, and U6-specific primers (Applied Biosystems) according to the manufacturer's instructions. Real-time PCR for miRNA quantification was carried out with TaqMan Universal Master Mix II (Applied Biosystems). The amplifications were conducted at $95^{\circ} \mathrm{C}$ for $10 \mathrm{~s}$ and at $60^{\circ} \mathrm{C}$ for $60 \mathrm{~s}$ using anAB7500 real-time PCR system (Applied Biosystems). Each sample was examined in triplicate and the amount of PCR product was normalized with respect to U6 as an internal control.

\section{Western Blot Analysis}

Prepared cells were harvested after washing with PBS. Collected cells were lysed with buffer $(50 \mathrm{mM}$ Tris-Cl $(\mathrm{pH}$ 7.5), $150 \mathrm{mM} \mathrm{NaCl}, 1 \mathrm{mM}$ EDTA (pH 8.0), 1\% Triton X-100, $1 \mathrm{mM}$ PMSF, $1 \mathrm{mM} \mathrm{Na} \mathrm{VO}_{4}$, and protease inhibitor cocktail (Roche Molecular Biochemicals, Indianapolis, IN, USA)). The same amount of protein was boiled at $95^{\circ} \mathrm{C}$ after adding SDS sample buffer (62.5 mM Tris-Cl (pH 6.8), 2\% SDS, 10\% glycerol, $\beta$-mercaptoethanol, and $0.002 \%$ bromophenol blue). Samples were loaded in 12\% SDS-PAGE gels for Bmi and Oct 4 and in $8 \%$ SDS-PAGE gels for Nestin, ABCG2, E-cadherin, Occludin, Vimentin, $\alpha$-SMA, TGF- $\beta$ receptor I, TGF- $\beta$ receptor II, Smad2, 3 , and $\mathrm{p}-\mathrm{Smad} 2,3$ and then transferred to PVDF membranes.

Antibodies used include the following: Oct4 (Santa Cruz Biotechnology, Santa Cruz, CA, USA; 1:1000), Bmi (Abcam, Cambridge, MA, USA; 1:1000), Nestin (Abcam; 1:1000), ABCG2 (Santa Cruz Biotechnology; 1:1000), E-cadherin (BD Bioscience; 1:1000), Occludin (Santa Cruz Biotechnology; 1:1000), Vimentin (Millipore, Bedford, MA, USA; 1:1000), 
Table 1 Primer sequences

\begin{tabular}{|c|c|c|}
\hline Gene & Direction & Sequence $\left(5^{\prime}-3^{\prime}\right)$ \\
\hline \multirow[t]{2}{*}{ CD44 } & $\mathrm{F}$ & GCTATTGAAAGCCTTGCAGAG \\
\hline & $\mathrm{R}$ & CGCAGATCGATTTGAATATAACC \\
\hline \multirow[t]{2}{*}{ Oct4 } & $\mathrm{F}$ & GACAACAATGAAAATCTTCAGGAGA \\
\hline & $\mathrm{R}$ & TTCTGGCGCCGGTTACAGAACCA \\
\hline \multirow[t]{2}{*}{ Bmi } & $\mathrm{F}$ & ATGTGTGTGCTTGTGGAG \\
\hline & $\mathrm{R}$ & AGTGGTCTGGTCTTGTGAAC \\
\hline \multirow[t]{2}{*}{ Nestin } & $\mathrm{F}$ & AACAGCGACGGAGGTCTCTA \\
\hline & $\mathrm{R}$ & TTCTCTTGTCCCGCAGACTT \\
\hline \multirow[t]{2}{*}{$A B C G 2$} & $\mathrm{~F}$ & CTGAGATCCTGAGCCTITGG \\
\hline & $\mathrm{R}$ & TGCCCATCACAACATCATCT \\
\hline \multirow[t]{2}{*}{ Vimentin } & $\mathrm{F}$ & GAGAGGAAGCCGAAAACACC \\
\hline & $\mathrm{R}$ & GCTTGGAAACATCCACATCG \\
\hline \multirow[t]{2}{*}{ E-cadherin } & $\mathrm{F}$ & TGCTTGGTTCACCAGTGGAT \\
\hline & $\mathrm{R}$ & TाTGTTGAGCAAGGCAACC \\
\hline \multirow[t]{2}{*}{$\alpha-S M A$} & $\mathrm{~F}$ & CCGACCGAATGCAGAAGGA \\
\hline & $\mathrm{R}$ & ACAGAGTATTTGGGTCCGAA \\
\hline \multirow[t]{2}{*}{ Occludin } & $\mathrm{F}$ & CTTCAGGCAGCCTCGTTACA \\
\hline & $\mathrm{R}$ & TCCCTGATCCAGTCCTCCTC \\
\hline \multirow[t]{2}{*}{ Smad7 } & $\mathrm{F}$ & CGAGAGTGGGGAGGCTCTAC \\
\hline & $\mathrm{R}$ & TTGTCCGAATTGAGCTGTCC \\
\hline \multirow[t]{2}{*}{$\beta$-Actin } & $\mathrm{F}$ & TTGCCGACAGGATGCAGAAG \\
\hline & $\mathrm{R}$ & AGGTGGACAGCGAGGCCAGG \\
\hline
\end{tabular}

$\alpha$-SMA(Abcam; $1: 1000)$, TGF- $\beta$ RI (Abcam; $1: 1000)$, TGF$\beta$ RII (Cell Signaling; 1:1000), Smad2 (Santa Cruz Biotechnology; 1:1000), Smad3 (Santa Cruz Biotechnology; 1:1000), p-Smad2/3 (Santa Cruz Biotechnology; 1:1000), and $\beta$-actin (Santa Cruz Biotechnology; 1:2000).

\section{Immunofluorescence Assay}

Cells were seeded on glass coverslips in six-well plates. After overnight incubation, the cells were washed with PBS three times, fixed in $4 \%$ formaldehyde for $10 \mathrm{~min}$, and permeabilized in $0.1 \%$ Triton X-100 in PBS for 3 min. The cells were washed three times with PBS and blocked with $1 \%$ BSA in PBS buffer. The cells were incubated with the primary antibodies overnight at $4{ }^{\circ} \mathrm{C}$. The slide was washed with PBS and incubated with FITC-conjugated secondary antibody for $1 \mathrm{~h}$ at room temperature and then mounted with DAPI. All samples were photographed using a Zeiss LSM700 confocal microscope (Carl Zeiss, Oberkochen, Germany).

\section{miRNA Microarray}

Total RNA of CD44(+) and CD44( - ) MKN45 cells was prepared using TRIzol reagent according to the manufacturer's instructions. Microarray was performed using the
Agilent Human miRNA 8 X $60 \mathrm{~K}$ (Rel 16.0 V2) platform (Agilent Technologies, Palo Alto, CA, USA). RNA hybridizations were performed with the Human microRNA Microarray Kit (Agilent Technologies) according to the manufacturer's protocol. Arrays were scanned on an Agilent $\mathrm{C}$ scanner. Images were quantified and data were processed using Agilent Feature Extraction Software (v 10.10.1.1). Raw data were extracted using the software provided by Agilent Feature Extraction Software (v 10.7.1.1). Selected miRNA TotalGeneSignal value was transformed by logarithm and normalized by the quantile method. The comparative analysis between test sample and control sample was carried out using fold-change. Hierarchical cluster analysis was performed using complete linkage and Euclidean distance as a measure of similarity.

\section{miRNA Inhibitor Transfection}

Cells were seeded into 6-well plates and incubated overnight. They were then transfected with miR-106b inhibitor and negative control (Applied Biosystems) at $30 \mathrm{nM}$ using Lipofectamine 2000 (Invitrogen) according to the manufacturer's instructions. After $72 \mathrm{~h}$, the cells were harvested and RNA and protein were isolated.

\section{Wound Healing Assay}

Cells were seeded in 24-well plates and incubated to attain $100 \%$ confluency. Linear scratches were made on the cell layers using pipette tips and washed with culture media. The cells were then incubated with serum-reduced medium for $72 \mathrm{~h}$. Gap closure was measured by light microscopy. Each sample was examined in triplicate.

\section{Invasion Assay}

Twenty-four-well, 8.0- $\mu \mathrm{m}$ Transwells (BD Biosciences) were coated with diluted Matrigel (BD Biosciences) in PBS and dried for $6 \mathrm{~h}$. The cells were resuspended in serum-free media, and then $4 \times 10^{5}$ cells were seeded in each top chamber. The lower chambers were filled with $10 \%$ FBS complete media for chemoattraction. To identify the effect of miR-106b knockdown, cells were transfected with miR-106b inhibitor and negative control. After $24 \mathrm{~h}$ the cells were seeded into the inserts. Cell invasion chambers were incubated for $48 \mathrm{~h}$ in a $37^{\circ} \mathrm{C}$ incubator supplemented with $5 \% \mathrm{CO}_{2}$. Non-invaded cells on the upper surface were removed with a cotton swab and the cells on the lower surface were fixed and stained with the Diff-Quik kit according to the manufacturer's instructions. Migrated cells on each insert were counted under a light microscope. Each sample was examined in triplicate.

\section{RESULTS}

CD44( + ) Cells Show Increased Sphere Formation and Upregulated Cancer Stem Cell Marker Expression

FACS-sorted CD44 $(+)$ cells and CD44(-) MKN45 cells (Figure 1a) were grown under serum-free conditions. After 
1-2 weeks of incubation, CD44 $(+)$ cells were found to generate more and larger spheroid colonies compared with CD44 $(-)$ cells. In addition, CD44(+) KATO III cells formed more than twice the number of spheres compared with CD44( - ) cells (Figure 1b). Real-time PCR was used to evaluate the mRNA level of stem cell markers in CD44 $(+)$ and CD44(-) MKN45 cells. Stem cell markers Oct4, Bmi, Nestin, and ABCG2 were analyzed. The mRNA level of all stem cell markers was upregulated in $\mathrm{CD} 44(+)$ cells compared with CD44( -$)$ cells. Western blot analysis was performed to identify protein expression of the corresponding markers. The stem cell markers were overexpressed in $\mathrm{CD} 44(+)$ cells in accordance with mRNA expression (Figure 1c). In addition, immunofluorescence assays showed a similar pattern of CD44 and Oct4 expression in fractionated cells (Figure 1d).

\section{CD44( + ) Cells Show Mesenchymal Characteristics Compared with $\operatorname{CD} 44(-)$ Cells}

Cancer stem cells have higher invasion and migratory capacity, which are thought to facilitate metastasis and growth. To compare the migration abilities of CD44( + ) and CD44( - ) cells, we conducted wound healing assay. Seventy-two hours after making gaps, $\mathrm{CD} 44(+)$ cells restored the wounds to a greater degree compared with CD44 $(-)$ cells. TheCD44 $(+)$ cells showed more than twice the migration length of CD44 ( - ) cells (Figure 2a). Cancer stem cells are thought to facilitate metastasis, through EMT characteristics related to the mobility of cells. We used well-known EMT markers: E-cadherin and Occludin as epithelial markers and Vimentin and $\alpha$-SMA ( $\alpha$-Smooth muscle actin) as mesenchymal markers. CD44 $(+)$ cells expressed higher levels of mesenchymal markers compared with CD44( -$)$ cells at both mRNA and
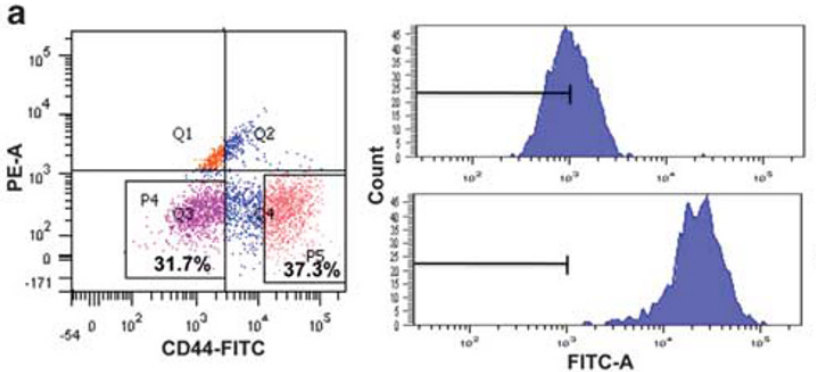

b
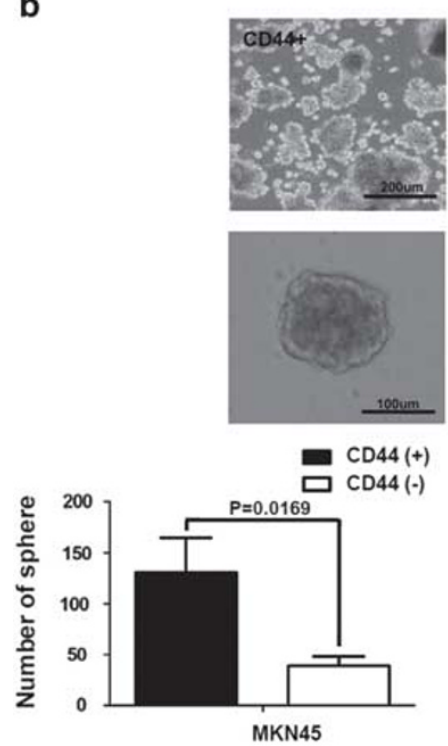
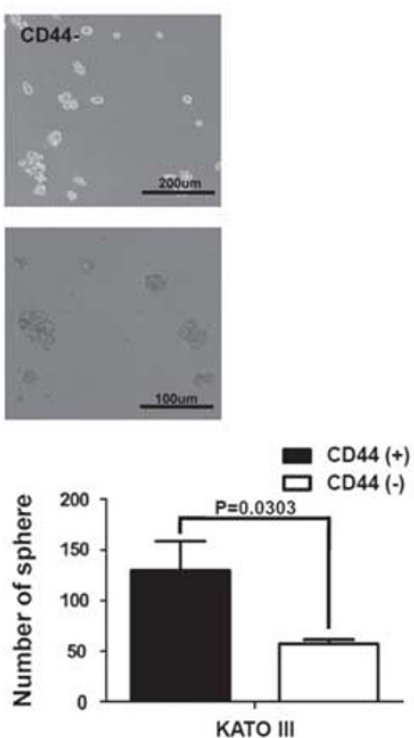

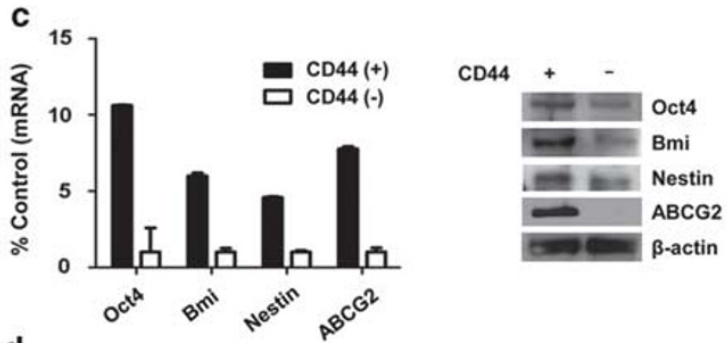

d
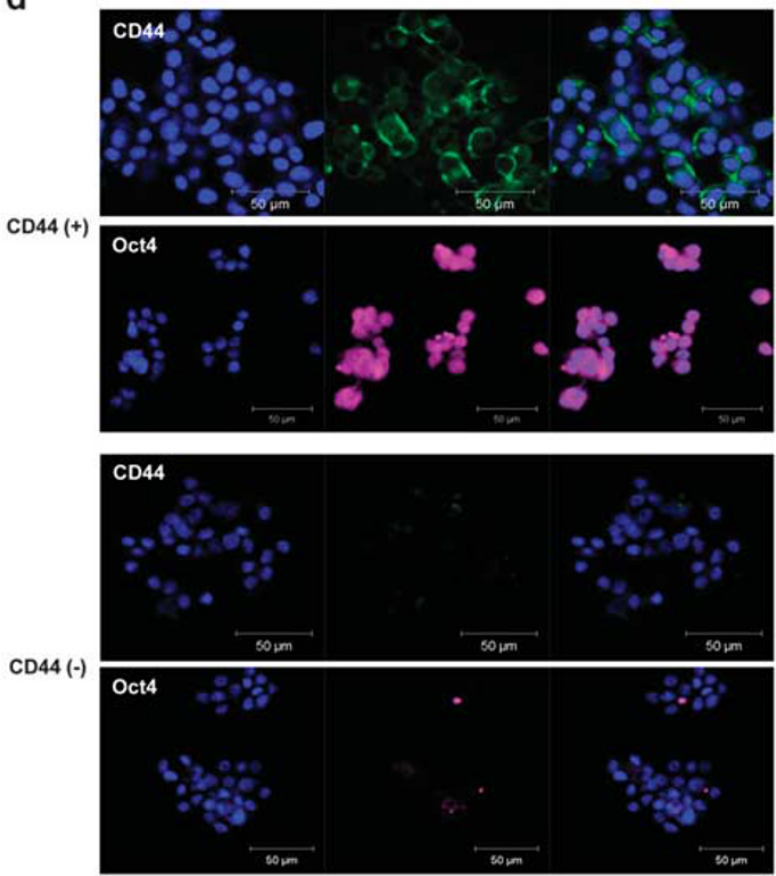

Figure $1 \mathrm{CD} 44(+)$ cells show increased sphere formation with upregulated stem cell marker expression compared with CD44( - ) cells. (a) CD44 expression analysis in MKN45 cells by FACS. After fractionating cells into CD44( + ) and CD44( - ), the degree of CD44 expression was analyzed by FACS. (b) Sphere formation abilities in CD44(+) cells and CD44( - ) MKN45 and KATO III cells were identified in a low-attachment plate with serum-free media, incubated for 1-2 weeks. The upper panel shows the photographs of spheres in CD44( +) and CD44( - ) MKN45 cells. The graphs show the average number of spheres in triplicate. (c) mRNA expression of cancer-stem-cell markers Oct4, Bmi, Nestin, and ABCG2 in CD44( +) and CD44( -) MKN45 cells was measured by real-time PCR (left panel). Protein expression of cancer stem cell markers in CD44( + ) cells and CD44( - ) cells was measured by western blot (right panel). (d) Expressions of CD44 and Oct4 were confirmed by immunofluorescence assay in CD44( + ) and CD44( - ) MKN45 cells with anti-CD44 (green, upper panel) and Oct4 (magenta, lower panel). The nucleus is stained with DAPI (blue). 
a

Oh

$72 \mathrm{~h}$
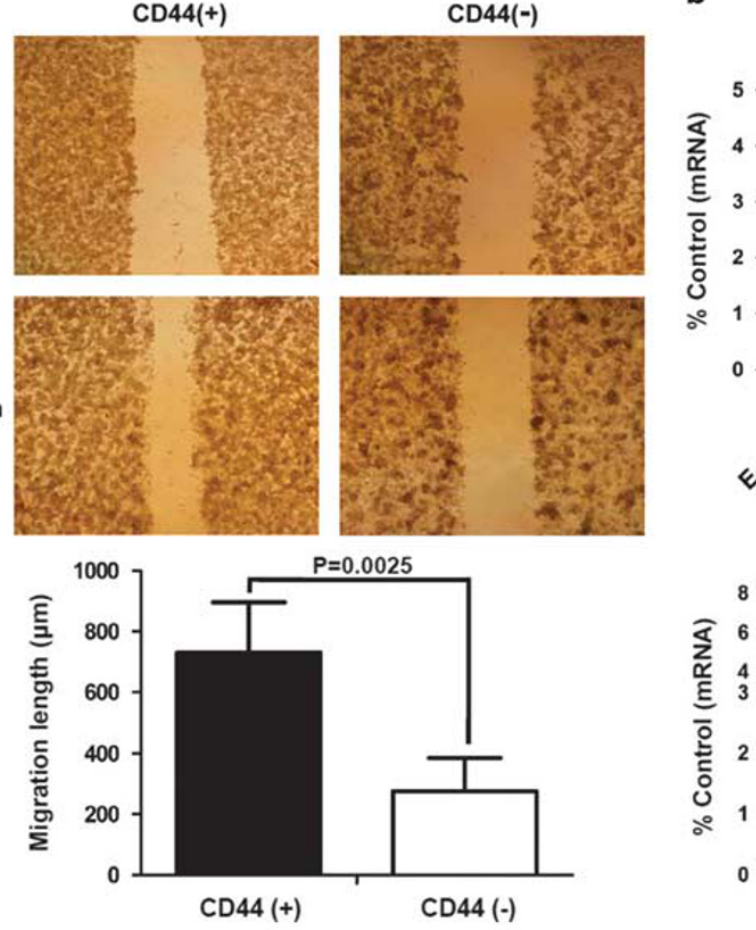

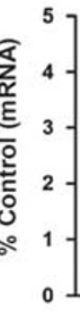

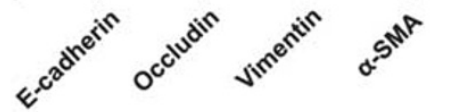

KATO III

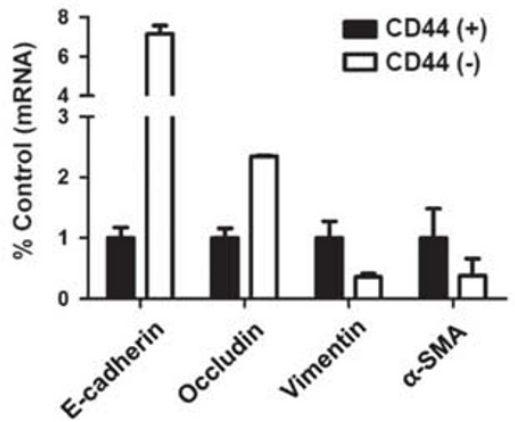

C

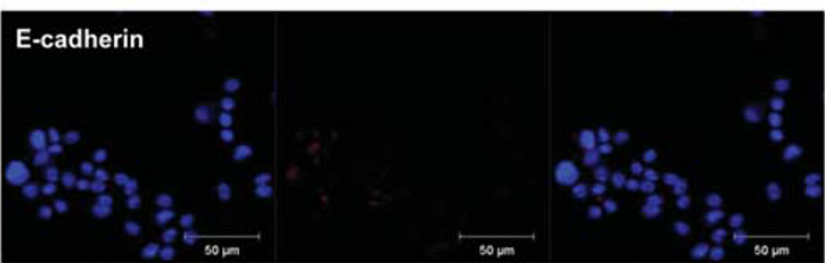

CD44 (+)
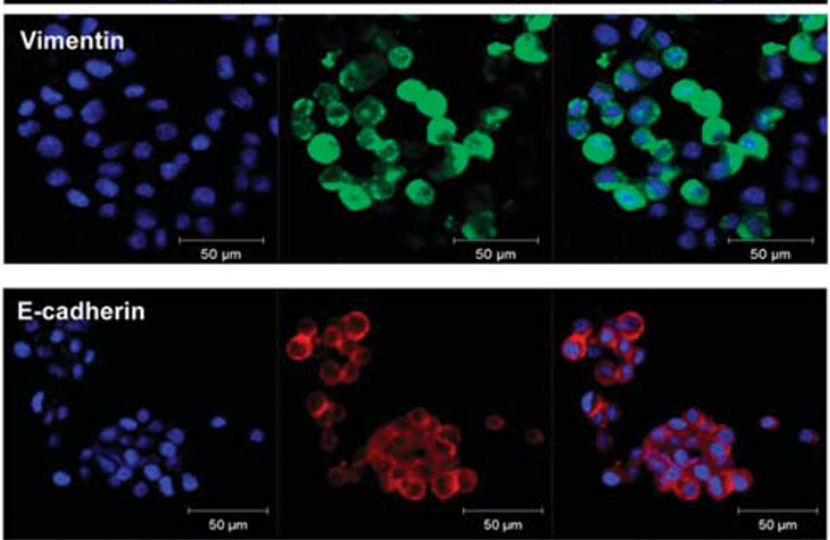

CD44 (-)

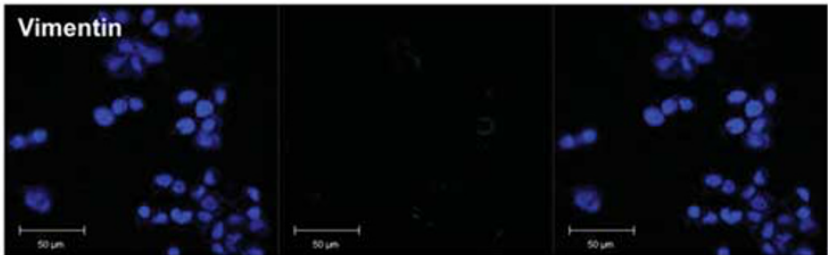


protein level. On the other hand, epithelial cell markers were downregulated in CD44 $(+)$ MKN45 and KATO III cells (Figure 2b). In immunofluorescence assays, we observed similar expression pattern of E-cadherin and Vimentin in CD44 $(+)$ and CD44 $(-)$ MKN45 cells (Figure 2c).

\section{CD44( + ) Cells Show Upregulation of the miR-106b Family in Microarray Analysis}

miRNA microarray analysis was performed on RNA isolated from both CD44 $(+)$ cells and CD44( - ) MKN45 cells in duplicate. Figure 3 a shows miRNAs that were upregulated in
CD44 $(+)$ cells as compared with CD44( - ) cells. We found that miR-106b, miR-93, and miR-25-the components of the miR-106b family-were upregulated in CD44 $(+)$ cells compared with CD44( - ) cells (Figure 3a). To validate the microarray data, miR-106b family levels were analyzed by real-time PCR on an independent set of RNA samples isolated from MKN45, KATO III, and SW480 cells matched for CD44 $(+)$ and CD44 $(-)$ cells (Figure 3b). We confirmed that the increased miR-106b family level in CD44 $(+)$ cells and the expression level of miR-106b showed the biggest difference between CD44(+) and CD44(-) cells among

a

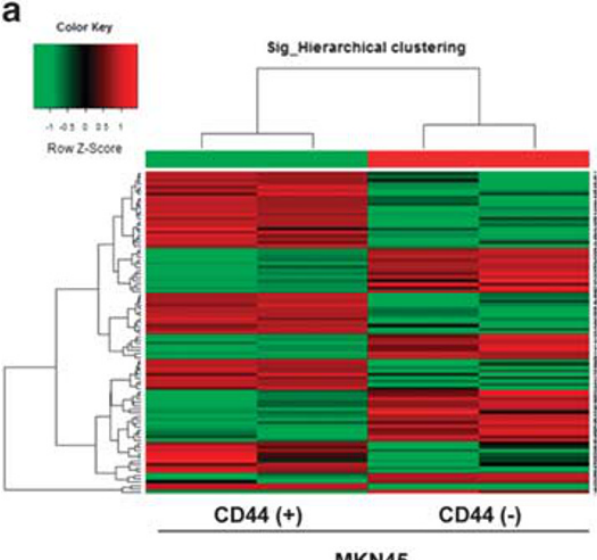

\begin{tabular}{lcc}
\multicolumn{3}{c}{ MKN45 } \\
\hline \multicolumn{1}{c}{ Gene name } & Fold change & p-value \\
\hline hsa-miR-634 & 3.7661 & 0.0482 \\
hsa-miR-193a-3p & 3.0942 & 0.0059 \\
hsa-miR-18b-3p & 2.5467 & 0.0186 \\
hsa-miR-425-5p & 2.0431 & 0.0014 \\
hsa-miR-221-3p & 2.0262 & 0.0186 \\
hsa-miR-424-5p & 1.9965 & 0.0057 \\
hsa-miR-93-5p & 1.7947 & 0.0033 \\
hsa-miR-15a-5p & 1.7935 & 0.0494 \\
hsa-miR-25-3p & 1.7799 & 0.0432 \\
hsa-miR-30e-5p & 1.7354 & 0.0430 \\
hsa-miR-210 & 1.6923 & 0.0420 \\
hsa-miR-30c-5p & 1.6847 & 0.0125 \\
hsa-miR-149-5p & 1.6161 & 0.0257 \\
hsa-miR-106b-5p & 1.6147 & 0.0350 \\
\hline
\end{tabular}

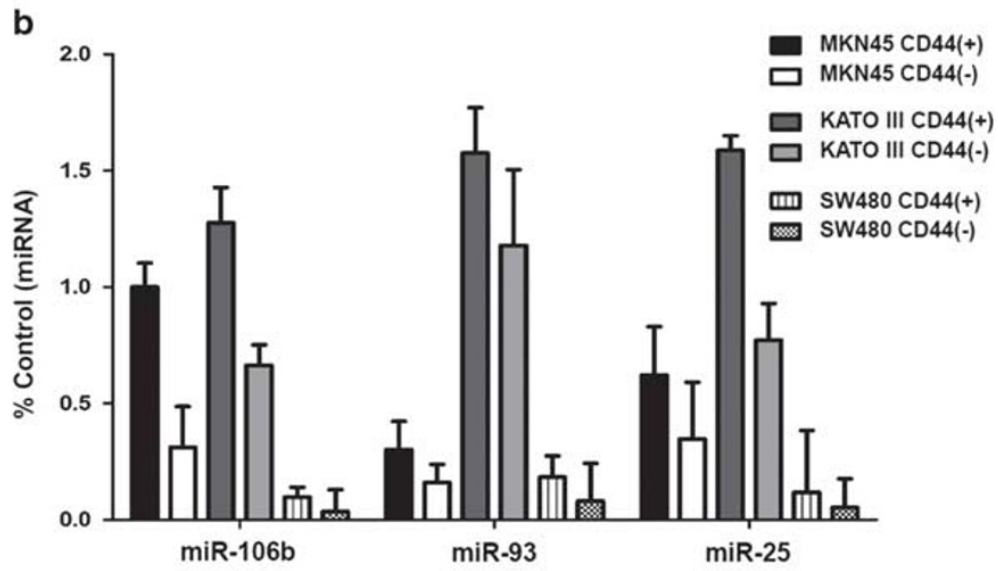

Figure 3 The miR-106b family is upregulated in CD44(+) cells. (a) Hierarchical clustering shows miRNA profiles in CD44(+) and CD44( - ) MKN45 cells. The colors indicate miRNA expression as exhibited in the color key. miRNAs upregulated in CD44( + ) cells are listed in the table (fold-change $>1.5$, $P<0.05$ ). (b) Expression of the miR-106b family was confirmed using real-time PCR in three cell lines. Cells were sorted according to CD44 expression by FACS.

Figure $2 \mathrm{CD} 44(+)$ cells show a higher rate of wound closure with upregulated EMT markers. (a) Cell migration capacity was identified by wound healing assay. The distance of migrations was measured in CD44(+) and CD44(-) MKN45 cells at $72 \mathrm{~h}$. The graph shows averages of migration distances in triplicate. (b) mRNA expression of EMT markers E-cadherin, Occludin, Vimentin, and $\alpha$-SMA in CD44( + ) and CD44( - ) MKN45 and KATO III cells was measured by real-time PCR that was normalized with respect to $\beta$-actin (left panel). Protein expression of EMT markers in CD44( + ) cells and CD44( - ) cells was measured by western blot (right panel). (c) Expressions of E-cadherin and Vimentin were confirmed by immunofluorescence assay in CD44 $(+)$ and CD44(-) MKN45 cells with anti-E-cadherin (red, upper panel) and Vimentin (green, lower panel). The nucleus is stained with DAPI (blue). 
these three miRNAs. This prompted us to carry out further study on the regulatory mechanism of miR-106b in gastric cancer stem-like cells.

\section{miR-106b regulates TGF- $\beta / S m a d$ signaling through the Inhibitory Protein Smad7}

miR-106b is known to activate the TGF- $\beta /$ Smad signaling pathway by targeting Smad7. Smad7 inhibits phosphorylation of Smad2/3 and suppresses the expression of the TGF- $\beta$ receptor as an inhibitor protein of Smad signaling. To examine the expression level of Smad7 and TGF- $\beta /$ Smad signal molecules, we performed real-time PCR and western blot in CD44(+) and CD44( -$)$ MKN45 cells. Smad7 was significantly decreased in CD44 $(+)$ cells at the mRNA and protein level (Figure $4 \mathrm{a}$ ). TGF- $\beta$ receptors and $\mathrm{p}-\mathrm{Smad} 2 / 3$, the active form of $S m a d 2 / 3$, was suppressed in CD44(-) cells as well. The downstream transcription factors Slug, Snail, and Twist were also upregulated in CD44(+) cells compared with CD44( - ) cells (Figure $4 \mathrm{~b}$ ).

\section{Expression of TGF- $\beta /$ Smad Signaling Molecules are Repressed by miR-106b Inhibition}

To study the function of miR-106b, we transfected miR-106b inhibitor and a negative control in CD44 (+) MKN45 cells. We conducted real-time PCR to confirm miRNA knockdown of miR-106b. miR-106b and miR-93 levels were decreased, but miR-25 remained unchanged (Figure 5a). Expression change of Smad7 by miR-106b downregulation was identified by real-time PCR and western blot. Inhibition of miR-106b induced increased Smad7 expression in CD44 $(+)$ cells (Figure 5b). The CD44 $(+)$ cell suppression of miR-106b expression by its inhibitor also showed decreased expression of TGF- $\beta$ receptor I, phosphorylated Smad2/3, and Slug. However, TGF- $\beta$ receptor II seems to be unaffected by miR-106b inhibition because Smad7 is known for regulating TGF- $\beta /$ Smad signaling by degrading TGF- $\beta$ receptor I, not TGF- $\beta$ receptor II. In addition, Smad7 blocked phosphorylation of $S m a d 2 / 3$ by inhibiting the recruitment of TGF- $\beta$ receptors (Figure 5c).

\section{Expression of Cancer Stem Cell Markers and Sphere Colony-Forming Ability were Suppressed by miR-106b Inhibition in CD44(+) Cells}

Sphere colony assay was conducted to validate the self-renewal capacity in CD44( + ) and CD44( + ) cells transfected with miR-106b inhibitor. After $24 \mathrm{~h}$, cells were transferred to ultra-low-attachment plates with serum-free media and incubated. miR-106b knockdown significantly decreased the sphere-forming ability in CD $44(+)$ cells (Figure 6a). The expression of cancer stem cell markers was measured by realtime PCR and western blot. mRNA and protein expressions of CD44 and cancer stem cell markers were relatively downregulated in the $\mathrm{CD} 44(+)$ cells transfected with miR-106b inhibitor compared with CD44 $(+)$ control cells (Figure 6b). The expression patterns of those markers in the a

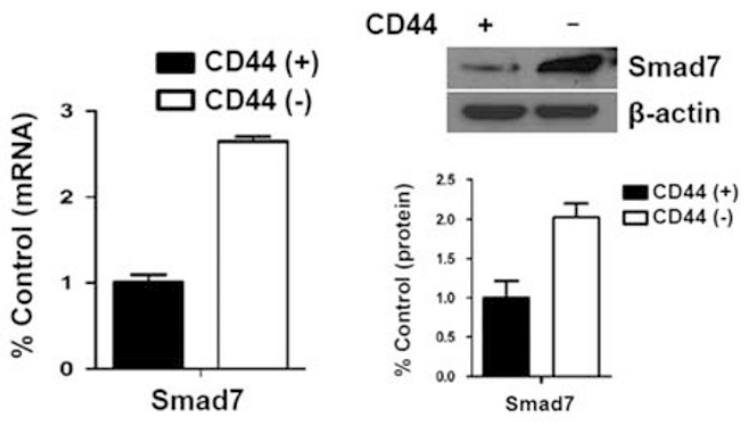

b
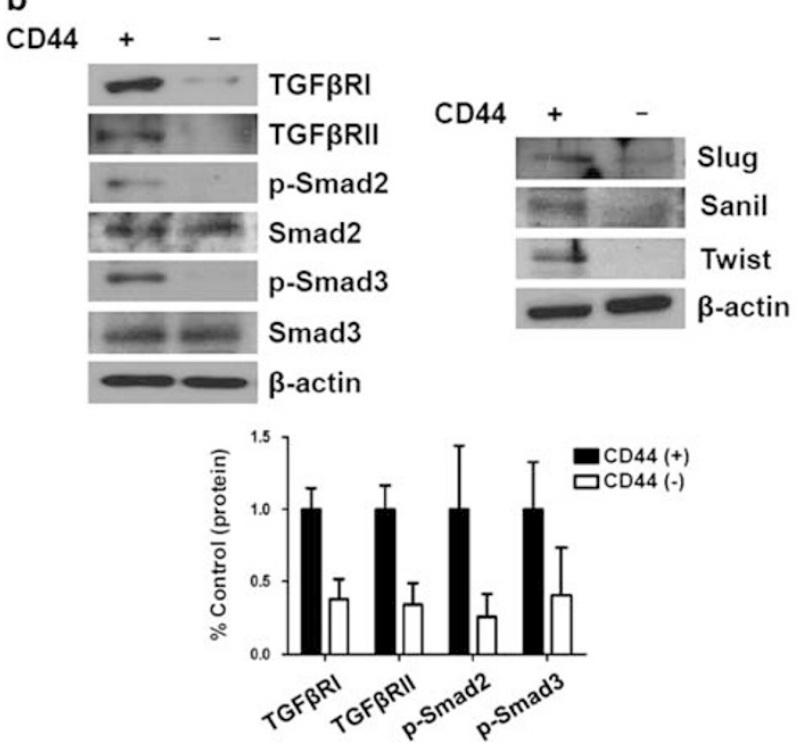

Figure 4 Downregulated Smad7 activates TGF- $\beta /$ Smad signaling molecules in CD44( + ) cells. (a) mRNA expression of Smad7 in CD44(+) and CD44( - ) MKN45 cells was measured by real-time PCR (left panel). Protein expression of Smad7 in CD44(+) and CD44(-) MKN45 cells was measured by western blot. The histogram displays the quantification of Smad7 protein levels as compared with $\beta$-actin control (right panel).(b) Protein expression of TGF- $\beta /$ Smad signaling molecules in CD44 $(+)$ and CD44( - ) MKN45 cells was measured by western blot, and the histograms display the quantification of Smad7 protein level as compared with $\beta$-actin control (left panel). Expressions of downstream genes of TGF- $\beta /$ Smad signaling were measured by western blot (right panel). (TGF $\beta$ RI, TGF- $\beta$ receptor I; TGF $\beta$ RII, TGF- $\beta$ receptor II).

$\mathrm{CD} 44(+)$ cells transfected with miR-106b inhibitor were similar to that of CD44( - ) cells. These results suggest that miR-106b affects the expression of stem cell genes and sphere formation ability in gastric cancer stem-like cells.

\section{EMT Markers and High Cellular Invasiveness are Inhibited by miR-106b Inhibition in CD44( + ) Cells}

An invasion assay was performed to identify phenotypic changes in cells with downregulated TGF- $\beta /$ Smad signaling by miR-106b inhibition. The number of invading cells in each group was counted and compared against the number of negative control cells by miR-106b inhibitor-transfected CD44 $(+)$ cells. The number of CD44 $(+)$ cells that are able 
a

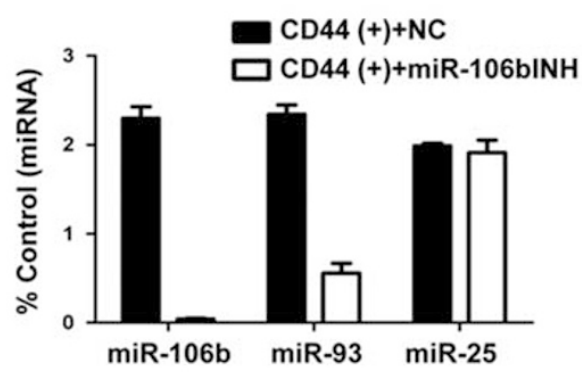

b
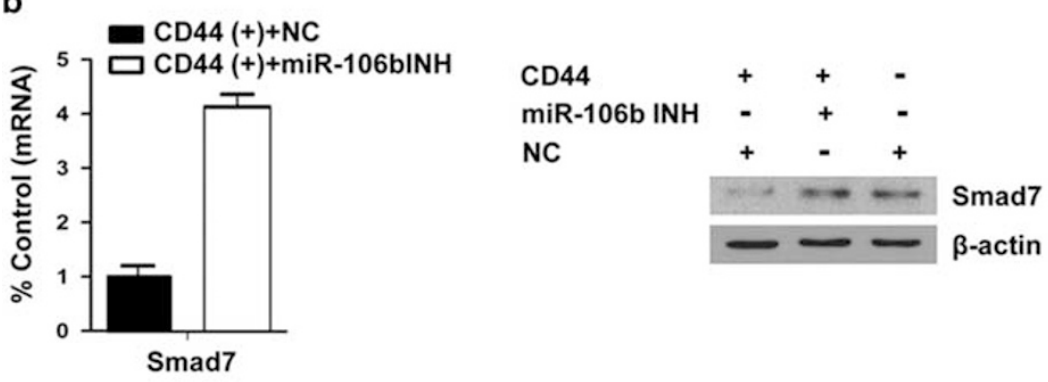

C
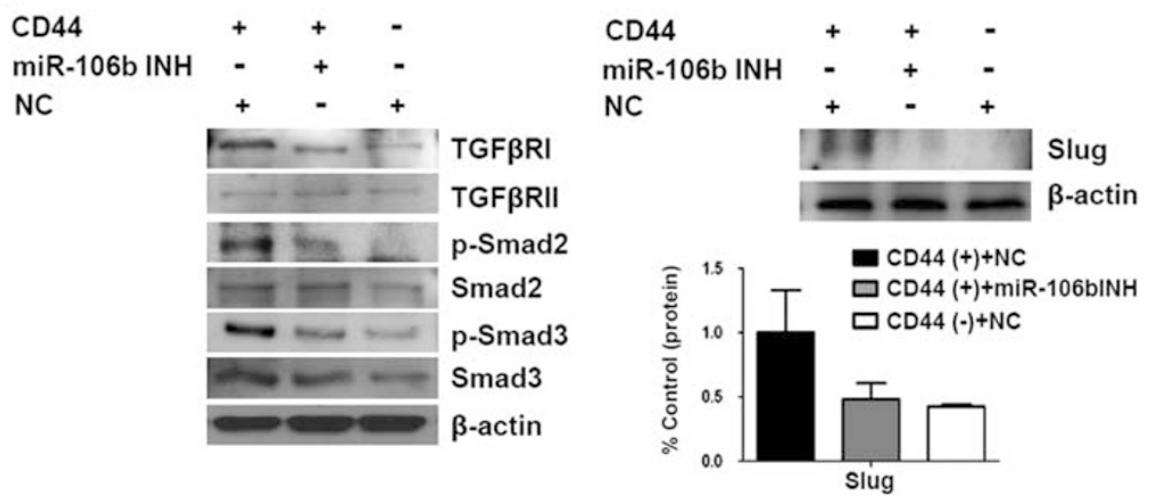

Figure 5 Inhibition of miR-106b expression suppresses TGF- $\beta /$ Smad signaling. (a) miRNA-106b family expression was identified by real-time PCR in CD44(+) MKN45 cells transfected with miR-106b inhibitor and negative control. (b) mRNA expression of Smad7 in CD44( +) MKN45 cells transfected with miR-106b inhibitor and negative control was measured by real-time PCR (left panel). Protein expressions of Smad7 in CD44( + ), CD44( - ) MKN45 cells transfected with negative control, and CD44(+) cells transfected with miR-106b inhibitor were measured by western blot (right panel). (c) Expression of TGF- $\beta /$ Smad signaling molecules was validated by western blot in CD44( + ), CD44( - ) MKN45 cells transfected with negative control, and $\mathrm{CD} 44(+)$ cells transfected with miR-106b inhibitor. The histogram displays the quantification of Slug protein level as compared with $\beta$-actin control. (NC, negative control; miR-106bINH, miR-106b inhibitor; TGF $\beta$ RI, TGF- $\beta$ receptor I; and TGF $\beta$ RII, TGF- $\beta$ receptor II).

to pass through the membrane was approximately twice that of $\mathrm{CD} 44(-)$ cells. Also, miR-106b inhibitor-transfected CD44 $(+)$ cells demonstrated significantly decreased invasion ability (Figure 7a). To identify EMT marker expression, EMT marker genes were analyzed. E-cadherin and Occludin were upregulated but Vimentin and $\alpha$-SMA were downregulated in miR-106b-downregulated $\mathrm{CD} 44(+)$ cells (Figure $7 \mathrm{~b}$ ).Taken together, miR-106b interference effectively suppresses mesenchymal gene expressions and the high invasiveness of CD44( + ) cells.

\section{DISCUSSION}

Many studies have confirmed the existence of cancer stem cells in various tumors in both cell lines and clinical samples.
Cancer stem cells can generate new tumors and can be a cause for drug resistance and tumor metastasis. Therefore, the elimination of cancer stem cells has attracted attention as a new approach of cancer therapy. ${ }^{21}$

In this study, in order to select the appropriate surrogate marker for stem cells, the widely used surrogate markers for stem cell surface markers, CD44 and EpCAM, were tested. Our result showed that $>99 \%$ of MKN45 and KATO III cell populations expressed EpCAM positivity (data not shown). We found that similar results regarding the EpCAM expressions in gastric cancer cell lines have been reported. ${ }^{22}$ As EpCAM expression cannot differentiate stem cell from gastric cancer cells, EpCAM was excluded from this study. On the other hand, the cells sorted by CD44 expression showed 

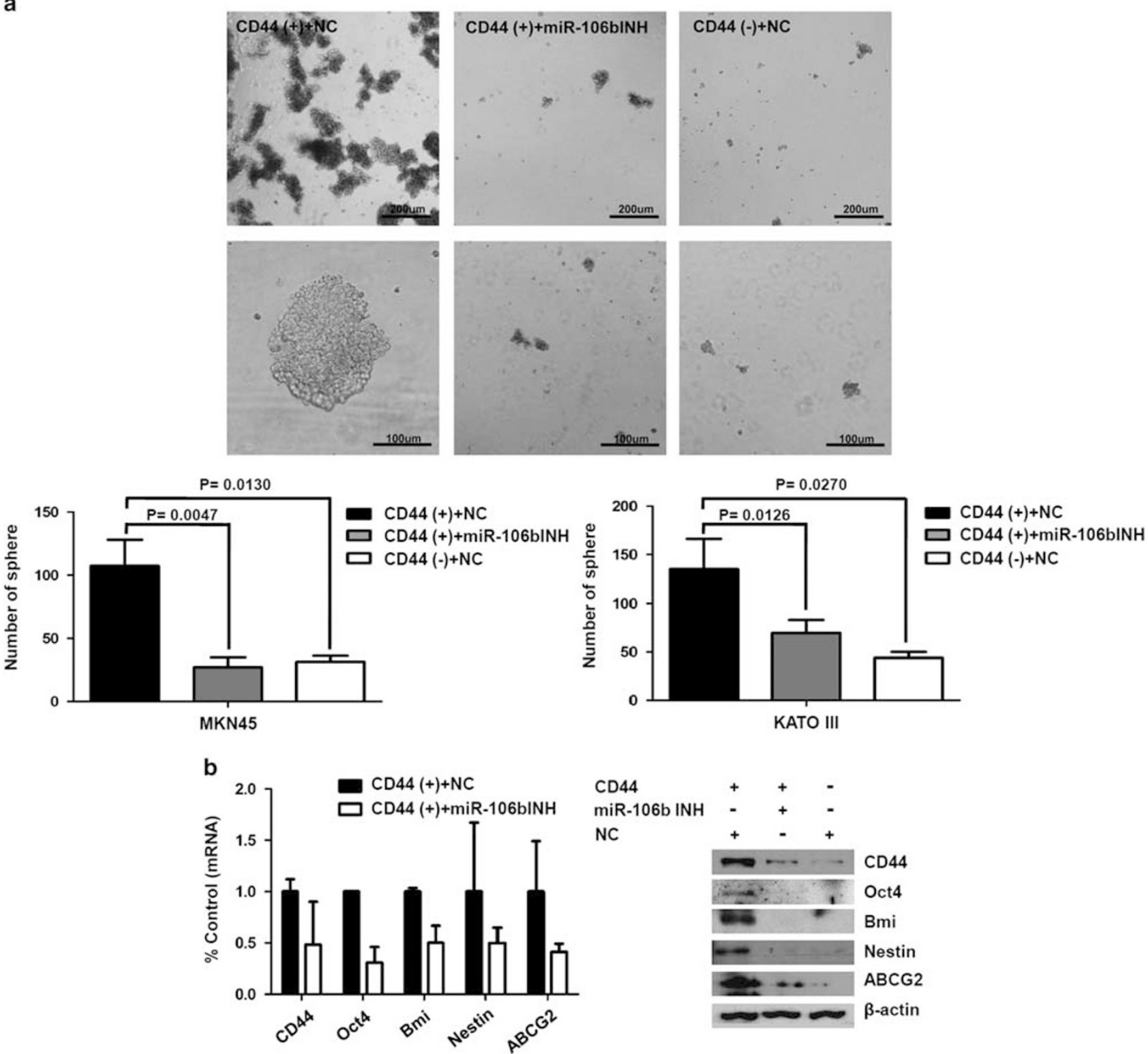

Figure 6 Inhibition of miR-106b expression suppresses the cancer stemness gene expression and sphere-forming ability in CD44( + ) cells. (a) Sphere formation abilities in CD44(+), CD44(-) MKN45, and KATO III cells transfected with negative control and CD44( +) cells transfected with miR-106b inhibitor were identified in a low-attachment plate with serum-free media and incubated for 1 week. The photographs show sphere colonies in MKN45 cells (upper panel). The graphs show the average number of spheres in triplicate (lower panel). (b) Expression of cancer stem cell markers CD44, Oct4, $B m i$, Nestin, and $A B C G 2$ was identified using real-time PCR and western blot in CD44(+), CD44(-) MKN45 cells transfected with negative control, and $\mathrm{CD} 44(+)$ cells transfected with miR-106b inhibitor. (NC, negative control; miR-106bINH, miR-106b inhibitor).

typical cancer stem cell phenotypes in gastric cancer cells. As a result, we used CD44 as a surrogate marker of gastric cancer stem-like cells in this study.

FACS-sorted CD44 $(+)$ cells showed many characteristics of cancer stem cells as compared with CD44(-) cells. The self-renewal capacity of the cells was examined by sphere formation ability, and $\mathrm{CD} 44(+)$ cells made more sphere colonies than CD44( - ) cells in MKN45 and KATO III cells. Also, the expression level of stem cell-related genes upregu- lated in many cancer stem cells was validated at the mRNA and protein level. Furthermore, EMT properties were compared in terms of molecular expression and phenotype between $\operatorname{CD} 44(+)$ and $\operatorname{CD} 44(-)$ cells. As a result, CD44( + ) cells showed a shift toward mesenchymal cell characteristics, as expected of cancer stem cells.

Next, we analyzed miRNA expression profiles in gastric cancer cells as a new approach to determining the regulatory mechanisms related to cancer stem cell characteristics. 
a
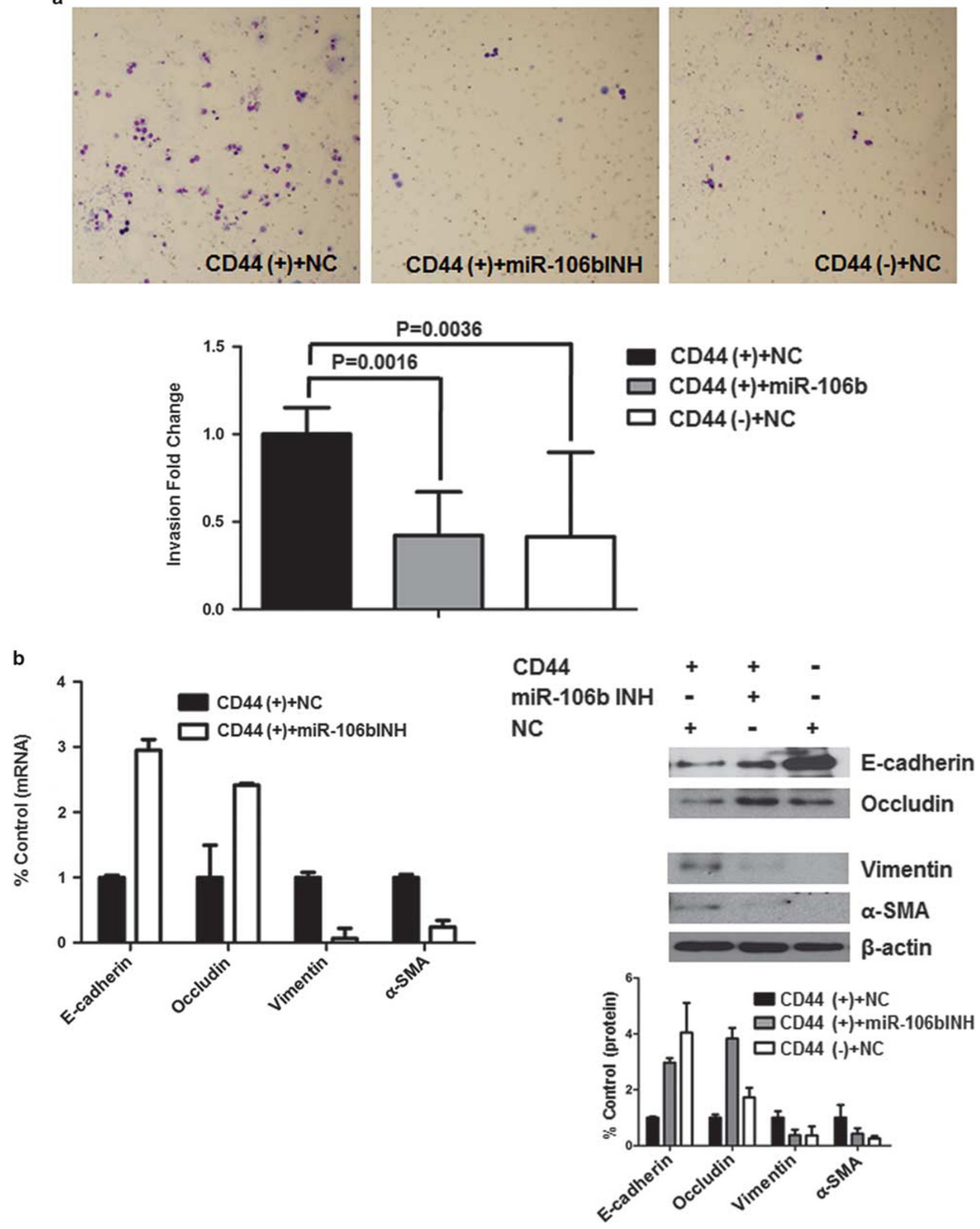

Figure 7 For caption please refer page 1380. 
Figure 7 Inhibition of miR-106b suppresses mesencymal marker gene expression and cell invasiveness. (a) Cell invasion ability was compared using Matrigel-coated invasion assay in CD44(+), CD44( - ) MKN45 cells transfected with negative control, and CD44( +) cells transfected with miR-106b inhibitor. Invaded cells were counted and converted to the graph. (b) Real-time PCR and western blot were conducted to investigate the expression of EMT markers E-cadherin, Occludin, Vimentin, and $\alpha-S M A$ in CD44(+), CD44( - ) MKN45 cells transfected with negative control, and CD44(+) cells transfected with miR-106b inhibitor. The histogram below the western blot images shows the quantification of protein levels as compared with $\beta$-actin control. (NC, negative control; miR-106bINH, miR-106b inhibitor).

$\mathrm{CD} 44(+)$ and $\mathrm{CD} 44(-)$ cells showed a different pattern of miRNA expression despite the two groups being of the same cell line. The miR-106b family composed of miR-106b, miR-93, and miR-25 was selected on the basis of miRNA microarray data. Although there were other miRNAs that showed increased expression in CD44 $(+)$ cells compared with CD44( - ) cells, we picked the miR-106b family because all three miRNAs were consistently upregulated in CD44 $(+)$ cells significantly. The miR-106b family is one of the upregulated miRNAs in high-grade cancer. ${ }^{23,24}$ The miR-106b family is located in intron 13 in the $\mathrm{Mcm} 7$ gene at chromosome $7 \mathrm{q} 22$. The family has two paralogs, miR-17-92 and miR-106a-363, which regulate cancer-related genes. This family has been studied mainly in cell cycle regulation. p21, the cyclin-dependent kinase inhibitor, is one of the target genes of the miR-106b family. miR-106b and miR-93 inhibit p21 expression by binding to its $3^{\prime} \mathrm{UTR}$, and miR-25 is known to suppress Bim. These actions of the miR-106b family may cause cancer stem cell proliferation in several tumors.

The TGF- $\beta$ signaling pathway has been identified as having a major role in many cellular pathways. Also TGF- $\beta$ signaling is implicated in tumor progression and cancer stem cell characteristics. ${ }^{25}$ In breast cancer, TGF- $\beta$ treatment causes the transition of $\mathrm{CD} 24^{+}$cells to $\mathrm{CD} 24^{-}$cells showing cancer stem cell characteristics. ${ }^{26}$ Activation of Smad2/3, TGF- $\beta$ signaling pathway transducers, maintains the pluripotency in human embryonic stem cells. ${ }^{27}$

Recently, miR-106b was also known to regulate EMT by the TGF- $\beta /$ Smad signaling pathway implicated in cancer stem-like cell development. ${ }^{25,28,29}$ The newly found target gene of miR-106b is Smad7, which acts as an inhibitor of TGF- $\beta /$ Smad signaling. Smad7 binds to TGF- $\beta$ receptor I and interrupts recruitment of TGF- $\beta$ receptors I and II. Smad7 degrades TGF- $\beta$ receptor I by interacting with ubiquitin ligase. ${ }^{30}$ Smad7 additionally blocks Smad2/3 activation by interfering with their phosphorylation. As a result, the downstream transcription factor of TGF- $\beta /$ Smad signaling, Slug, is downregulated. ${ }^{31}$

In this study, $\mathrm{CD} 44(+)$ gastric cancer stem-like cells, with upregulated miR-106b, showed suppressed expression of Smad7 as compared with CD44 $(-)$ cells. We also observed activated TGF- $\beta /$ Smad signaling and increased expression of Slug in CD44 $(+)$ cells, and conducted miR-106b inhibitior transfection in $\mathrm{CD} 44(+)$ cells to confirm the regulatory mechanism of miR-106b. Inhibition of miR-106b caused increased Smad7 expression and suppressed the TGF- $\beta /$ Smad signaling pathway, and it led to inhibition of EMT and stem cell characteristics. Cancer stemness genes were downregulated and self-renewal capacity was inhibited in CD44 $(+)$ cells by miR-106b knockdown. Also, miR-106b inhibition repressed the upregulated mesenchymal characteristics of CD44 $(+)$ cells.

In our data, expression of miR-106b and CD44 seems to be connected. Based on this, we guess that CD44 expression is correlated with miR-106b expression. However, we have not confirmed the mechanism of how they affect each other. We are carrying out experiments on this and it would be a main theme in our next paper.

Taken together, our results indicate that miR-106b is upregulated in CD44(+) gastric cancer stem-like cells, and the cancer stem cell characteristics are retained by modulation of the TGF- $\beta /$ Smad signaling pathway. miR-106b can be a new marker of gastric cancer stem cells and may be a promising target for cancer treatment in gastric cancer.

\section{ACKNOWLEDGMENTS}

The present study was supported by the Basic Science Research Program through the National Research Foundation of Korea (NRF) funded by the Ministry of Education, Science and Technology (Grant Number NRF-2010-0010739, NRF-2013R1A1A2009707).

\section{DISCLOSURE/CONFLICT OF INTEREST}

The authors declare no conflict of interest.

1. Ferlay J, Shin HR, Bray F, et al. Estimates of worldwide burden of cancer in 2008: GLOBOCAN 2008. Int J Cancer 2010;127:2893-2917.

2. Jemal A, Center MM, DeSantis C, et al. Global patterns of cancer incidence and mortality rates and trends. Cancer Epidemiol Biomarkers Prev 2010;19:1893-1907.

3. Jordan CT, Guzman ML, Noble M. Cancer stem cells. New Engl J Med 2006;355:1253-1261.

4. Bonnet D, Dick JE. Human acute myeloid leukemia is organized as a hierarchy that originates from a primitive hematopoietic cell. Nat Med 1997;3:730-737.

5. Singh SK, Clarke ID, Terasaki M, et al. Identification of a cancer stem cell in human brain tumors. Cancer Res 2003;63:5821-5828.

6. Al-Hajj $M$, Wicha MS, Benito-Hernandez $A$, et al. Prospective identification of tumorigenic breast cancer cells. Proc Natl Acad Sci 2003;100:3983-3988.

7. Takaishi S, Okumura T, Tu S, et al. Identification of gastric cancer stem cells using the cell surface marker CD44. Stem Cells 2009;27: 1006-1020.

8. Zhang C, Li C, He F, et al. Identification of CD44+CD24+ gastric cancer stem cells. J Cancer Res Clin 2011;137:1679-1686.

9. Rocco A, Compare D, Nardone G. Cancer stem cell hypothesis and gastric carcinogenesis: experimental evidence and unsolved questions. World J Gastrointest Oncol 2012;4:54-59.

10. Thiery JP. Epithelial-mesenchymal transitions in tumour progression. Nat Rev Cancer 2002;2:442-454. 
11. Mani SA, Guo W, Liao M-J, et al. The epithelial-mesenchymal transition generates cells with properties of stem cells. Cell 2008;133:704-715.

12. Bartel DP. MicroRNAs: genomics, biogenesis, mechanism, and function. Cell 2004;116:281-297.

13. Shimono $Y$, Zabala M, Cho RW, et al. Downregulation of miRNA-200c links breast cancer stem cells with normal stem cells. Cell 2009;138: 592-603.

14. Liu C, Kelnar K, Liu B, et al. The microRNA miR-34a inhibits prostate cancer stem cells and metastasis by directly repressing CD44. Nat Med 2011;17:211-215.

15. Guo J, Miao Y, Xiao B, et al. Differential expression of microRNA species in human gastric cancer versus non-tumorous tissues. J Gastroen Hepatol 2009;24:652-657.

16. Kim YK, Yu J, Han TS, et al. Functional links between clustered microRNAs: suppression of cell-cycle inhibitors by microRNA clusters in gastric cancer. Nucleic Acids Res 2009;37:1672-1681.

17. Ambs S, Prueitt RL, Yi M, et al. Genomic profiling of microRNA and messenger RNA reveals deregulated microRNA expression in prostate cancer. Cancer Res 2008;68:6162-6170.

18. Hayashita $\mathrm{Y}$, Osada $\mathrm{H}$, Tatematsu $\mathrm{Y}$, et al. A polycistronic microRNA cluster, miR-17-92, is overexpressed in human lung cancers and enhances cell proliferation. Cancer Res 2005;65:9628-9632.

19. Ivanovska I, Ball AS, Diaz RL, et al. MicroRNAs in the miR-106b family regulate $\mathrm{p} 21 / \mathrm{CDKN} 1 \mathrm{~A}$ and promote cell cycle progression. Mol Cell Biol 2008:28:2167-2174.

20. Petrocca $F$, Vecchione $A$, Croce CM. Emerging role of miR-106b-25/ miR-17-92 clusters in the control of transforming growth factor $\beta$ signaling. Cancer Res 2008;68:8191-8194.

21. Guo W, Lasky JL, Wu H. Cancer stem cells. Pediatr Res 2006;59: 59R-64R.
22. Nishikawa $S$, Konno $M$, Hamabe $A$, et al. Aldehyde dehydrogenase high gastric cancer stem cells are resistant to chemotherapy. Int J Oncol 2013:42:1437-1442.

23. Blenkiron C, Goldstein LD, Thorne NP, et al. MicroRNA expression profiling of human breast cancer identifies new markers of tumor subtype. Genome Biol 2007;8:R214.

24. Rothe $\mathrm{F}$, Ignatiadis $\mathrm{M}$, Chaboteaux $\mathrm{C}$, et al. Global microRNA expression profiling identifies MiR-210 associated with tumor proliferation, invasion and poor clinical outcome in breast cancer. PLoS One 2011;6:e20980.

25. Singh A, Settleman J. EMT, cancer stem cells and drug resistance: an emerging axis of evil in the war on cancer. Oncogene 2010;29: 4741-4751.

26. Morel A-P, Lièvre $M$, Thomas $C$, et al. Generation of breast cancer stem cells through epithelial-mesenchymal transition. PloS One 2008;3:e2888.

27. James $D$, Levine AJ, Besser $D$, et al. TGF $\beta$ /activin/nodal signaling is necessary for the maintenance of pluripotency in human embryonic stem cells. Development 2005;132:1273-1282.

28. Smith A, Iwanaga $R$, Drasin D, et al. The miR-106b-25 cluster targets Smad7, activates TGF- $\beta$ signaling, and induces EMT and tumor initiating cell characteristics downstream of Six 1 in human breast cancer. Oncogene 2012;31:5162-5171.

29. Yau WL, Lam CSC, Ng L, et al. Over-expression of miR-106b promotes cell migration and metastasis in hepatocellular carcinoma by activating epithelial-mesenchymal transition process. PloS One 20138.

30. Ebisawa T, Fukuchi M, Murakami G, et al. Smurf1 interacts with transforming growth factor- $\beta$ type I receptor through Smad7 and induces receptor degradation. J Biol Chem 2001;276:12477-12480.

31. Nakao A, Afrakhte M, Morn A, et al. Identification of Smad7, a TGF $\beta$ inducible antagonist of TGF- $\beta$ signalling. Nature 1997;389:631-635. 